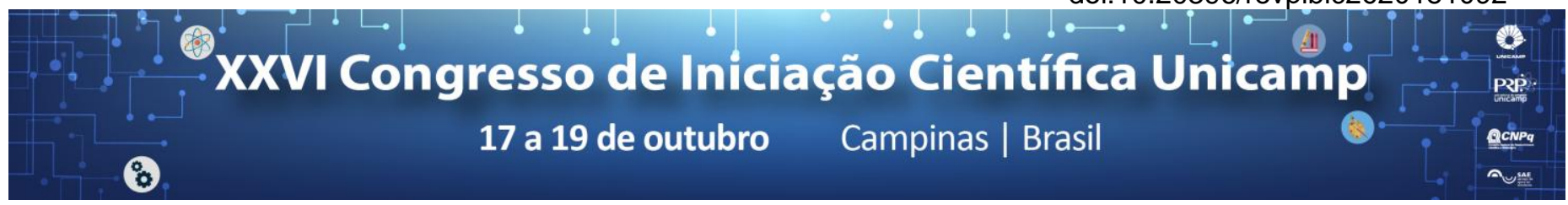

\title{
Base formulation prospection of a colloidal beverage mimic system added with lutein
}

\author{
Nicholas H. P. Taveira*, Mayara S. Ponte, Lilian R. B. Mariutti.
}

\begin{abstract}
Lutein daily intake is related to the maintenance of visual and cognitive health. The incorporation of this lipophilic compound into an aqueous matrice requires the use of an emulsion as a carrier system. Lutein was extracted from marigold petals by supercritical fluid extraction, using sunflower seed as co-solvent at 3 different temperatures. Extraction efficiency was evaluated through the total carotenoid content. A colloidal beverage mimic system was prepared with an oil-in-water emulsion formulated with carotenoid extracts, and it was preliminarily observed that there was degradation of the carotenoids during 15 days of refrigerated storage.
\end{abstract}

Key words: Carotenoids, supercritical extraction, emulsion

\section{Introduction}

The daily intake of lutein can lead to the accumulation of this carotenoid in both eye and brain tissues, which supports a role for lutein in the protection of visual and cognitive health ${ }^{1}$. The use of lutein to prevent degenerative diseases becomes an interesting alternative, especially added to dairy beverages, which are one of the main products for addition of functional compounds. However, a technical difficult is faced to incorporate carotenoids, which are lipophilic compounds, in a water based matrices, so, it is necessary to use carrier systems, such as emulsions, to facilitate the addition of these compounds in this type of product. Carotenoids are conventionally extracted by organic solvents, but the residues originated from this type of extraction may cause environmental problems. In face of this, there has been an interest in alternative technologies such as supercritical fluid extraction ${ }^{2}$. In this work, we presented the base formulation of a colloidal beverage mimic system and the quantification of the total carotenoids of the beverage and of the supercritical extracts added in its preparation.

\section{Results and Discussion}

Carotenoid was obtained from marigold petals through extraction with supercritical fluid, using $\mathrm{CO}_{2}$ and sunflower seed as solvent and co-solvent, respectively. Three temperature conditions were evaluated $\left(30^{\circ} \mathrm{C}\right.$, $40^{\circ} \mathrm{C}$ and $60^{\circ} \mathrm{C}$ ). The extracts were characterized by their carotenoid content, spectrophotometrically quantified (Chart 1). The extracts obtained at $30^{\circ} \mathrm{C}$ and $40^{\circ} \mathrm{C}$ were used to prepare the emulsion because of their higher lutein content, since higher temperatures resulted in a decrease in total carotenoid content.

Chart 1. Carotenoid content (mg lutein/g extract).

\begin{tabular}{|c|c|}
\hline Extract & Carotenoid content \\
\hline $30^{\circ} \mathrm{C}$ & $0.57 \pm 0.12$ \\
\hline $40^{\circ} \mathrm{C}$ & $0.52 \pm 0.06$ \\
\hline $60^{\circ} \mathrm{C}$ & $0.42 \pm 0.24$ \\
\hline
\end{tabular}

The oil-in-water emulsion was prepared by microfluidization (3 cycles, 10000 psi) with $6.5 \mu \mathrm{g}$ lutein $/ \mathrm{mL}, 5 \%$ sunflower oil and $1.5 \%$ whey protein isolate in aqueous solution.

The colloidal beverage mimic system was prepared with an isotonic beverage base containing: citric acid, sodium benzoate, sodium citrate, potassium chloride, sodium chloride, potassium phosphate monobasic, fructose, glucose, whey protein isolate and sucrose, besides $17 \%$ oil-in-water emulsion. The mixture was prepared under magnetic stirring, followed by homogenization in Ultraturrax T25.

For the beverage, quantification of carotenoids was carried out after 15 days of refrigerated storage $\left(4^{\circ} \mathrm{C}\right)$. Briefly, methanol was added to the sample, followed by freezing, centrifugation and the upper layer was discarded for removal of non-carotenoid lipid components that hinder the subsequent extraction phases. After that, ethyl ether, hexane and $10 \% \mathrm{NaCl}$ were added, followed by centrifugation $^{3}$. The procedure was repeated until exhaustive extraction and the carotenoid content was determined spectrophotometrically. The beverage had $0.84 \pm 0.06 \mu \mathrm{g}$ lutein $/ \mathrm{g}$, with $71 \%$ of recovery related to the total carotenoid content added in its preparation. Due to the storage time, carotenoid degradation is considered to occur.

\section{Conclusions}

For the project continuity the supercritical extract obtained at the lowest temperature $\left(30^{\circ} \mathrm{C}\right)$ was chosen to avoid the degradation of the carotenoids and to present a higher content of total carotenoids. Stability tests will be needed to evaluate and prevent carotenoid degradation and increase the shelf life of the beverage.

\section{Acknowledgement}

CNPq (Projeto Universal-455748/2014-4), N.H.P.T. Bolsa IC Faepex-Unicamp (Proc. 3057/17) e M.S.P. Bolsa Mestrado Faepex-Unicamp (3314/17).

${ }^{1}$ Johnson, E. Nutr. Rev. 2014, 72, 605

${ }^{2}$ Gao, Y.; Liu, X.; Xu, H. Zhao, J.; Wang, Q.; Liu, G. e Hao, Q. Sep. Purif. Technol. 2010, 71, 214.

${ }^{3}$ Ríos, J.J.; Xavier, A.A.O.; Díaz-Salido, E.; Arenilla-Vélez, I.; Jarén-Galán, M.; Garrido-Fernández, J.; Aguayo-Maldonado, J.; Pérez-Gálvez, A. Mol. Nutr. Food Res. 2017, 61. 